\title{
Therapy-Induced Changes of Gene Expression in a Matched Pair of Small Cell Lung Cancer (SCLC) Cell Lines
}

\author{
Gerhard Hamilton, Ernst Ulsperger, Klaus Geissler, Ulrike Olszewski
}

Ludwig Boltzmann Cluster of Translational Oncology, Vienna, Austria.

Email: gerhard.hamilton@toc.lbg.ac.at

Received July $31^{\text {st }}, 2012$; revised September $5^{\text {th }}, 2012$; accepted September $23^{\text {rd }}, 2012$

\begin{abstract}
Extended stage small cell lung cancer (SCLC) responds to platinum/vepeside-based first-line chemotherapy but relapses early as drug-resistant tumor associated with a dismal prognosis. A pair of SCLC cell lines obtained from a single patient at different time points during treatment allows for the investigation of the changes in gene expression before (GLC14) and following cycles of chemotherapy and irradiation (GLC19). GLC19 cells were reported to reveal an increased doubling time and exhibit increased chemoresistance to doxorubicin, etoposide, melphalan and vinblastine. Upregulated transcripts in GLC19, as assessed by microarray analysis, comprised genes involved in regulation of cellular growth (NGFRAP1/BEX3), adhesion, glutathione metabolism and, in particular, WNT/Notch pathways and the putative cancer stem cell phenotype (CD44, ALDH1A1, and AKR1C1/13). Metallothioneins, tubulins TUBA3/4 and tumor protein p53 inducible protein 11 (TP53IP11) were downregulated in this cell line compared to GLC14. Except increased expression of glutathione transferases no classical markers of chemoresistance were found, pointing to a role of altered growth control/differentiation and reduced accessibility of these SCLC cells that grow as multicellular spheroids. In conclusion, treatment of this single SCLC patient with cyclophosphamide, doxorubicin and etoposide (CDE) followed by radiotherapy ultimately enriched tumor cells that display the typical signature of tumor-initiating cells/cancer stem cells (CICs/CSCs).
\end{abstract}

Keywords: Small Cell Lung Cancer; GLC14; GLC19; Chemotherapy; Chemoresistance; Gene Expression; Cancer-Initiating Cells

\section{Introduction}

Due to over one million cases diagnosed every year and corresponding low survival rates, lung cancer is still the leading cause of cancer death despite a lower incidence compared to other tumor entities [1]. Small cell lung cancer (SCLC) represents approximately $13 \%$ of all lung cancer diagnoses and its incidence has reduced over the last 20 years [2]. These highly malignant neuroendocrine tumors of the lung originate from neuroendocrine cells (Amine Precursor Uptake and Decarboxylation/APUD cells) in the bronchus called Feyrter cells. Treatment of SCLC has remained challenging because of rapid growth, early dissemination and development of drug resistance during the course of the disease [3]. The predominant risk factor for SCLC is cigarette smoking with smokers facing a 20 - 30-fold higher incidence of SCLC than non-smokers [2]. Although the number of cases of SCLC is declining in man the frequency is rising in women due to increased use of tobacco. Within the context of the next 20 years, the incidence of lung cancer will grow with an increasing number of cases unrelated to smoking [4].

Without treatment SCLC has the most aggressive clinical course of any type of pulmonary tumors, with median survival from diagnosis of only 2 - 4 months [2]. Compared with other types of lung cancer, SCLC has a greater tendency to be widely disseminated at time of diagnosis while being much more responsive to chemotherapy and radiation therapy first-line. Because patients with SCLC tend to develop distant metastases, localized forms of treatment, such as surgical resection or radiation therapy, rarely produce long-term survival. With current chemotherapy regimens survival is unequivocally prolonged; however, the overall survival at 5 years is only $5 \%-10 \%[2,5]$. At time of diagnosis approximately $30 \%$ of patients with SCLC have a tumor confined to either the hemithorax of origin, the mediastinum, or the supraclavicular lymph nodes. These patients are classified as having limited-stage disease and 
hence a median survival of 16 - 24 months with current forms of treatment can reasonably be expected $[1,5]$. Patients with tumors that have spread beyond the supraclavicular areas are categorized as having extensive-stage disease and reveal a worse prognosis than patients with limited-stage disease. In these cases a median survival of 6 - 12 months is reported with currently available therapy, whereas long-term disease-free survival is rare.

The majority of patients with SCLC die of their tumor despite the best available treatment $[5,6]$. Chemotherapy improves the survival of patients with limited-stage or extensive-stage SCLC, but is curative in only a minority of patients. In patients with limited-stage SCLC combination chemotherapy produces results that are clearly superior to single-agent treatment. Current regimens yield overall objective response rates of $65 \%-90 \%$ and complete response rates of $45 \%-75 \%$. The combination of etoposide and cisplatin chemotherapy with concurrent chest radiation therapy has now been used in various single institutional studies and achieved median survival rates of 18 - 24 months and a $40 \%$ - 50\% 2-year survival with less than $3 \%$ treatment-related mortality $[1,5,7]$. The prognosis for patients with SCLC that has progressed despite chemotherapy is exceptionally poor regardless of the stage, with expected median survival of 2 - 3 months. These patients should be considered for palliative therapy or clinical trials. Though no single chemotherapy regimen should be regarded standard, those that have shown activity as second-line treatment include oral etoposide, etoposide/cisplatin, cyclophosphamide/ doxorubicin/vincristine (CAV), lomustine/methotrexate, paclitaxel and topotecan [3,5]. Pharmacological inhibittors of receptor tyrosine kinases, including c-Kit, epidermal growth factor receptor (EGFR), insulin-like growth factor-I receptor (IGFR1) and vascular endothelial growth factor receptor (VEGFR), have been investigated as potential antitumor agents in SCLC with disappointing results $[5,8]$. Thus, the task of identifying targeted agents with clinically meaningful activity has so far proven to be less successful than in other tumors.

In summary, the outcome of treatment of SCLC is characterized by a lack of progress and extremely short survival rates in advanced disease exhibiting little improvement in the past decades. Despite high initial response rates the majority of patients relapse and die of their disease. Many suggestions discussed the reasons for this failure, including the presence of resistant stem cells, development of acquired resistance, redundancy in signaling pathway etc., while the definite underlying cause has remained unclear. Translational research to correlate cellular target pathway activation/inhibition with clinical endpoints is essential to maximize the knowledge gained from trials and to identify potential predictive markers. Longitudinal biopsies, viable cells or cell lines that re- flect the course of chemosensitivity towards resistant tumors are rarely available for SCLC patients. However, a series of three tumor biopsies with corresponding cell lines derived thereof, namely GLC14, GLC16 and GLC19, was described for a single SCLC patient by Berendsen et al. in 1988 [9]. There was a good accordance in the morphological, biochemical, and immunohistological findings between the cell lines compared to those obtained in the biopsies This tumor progressed from an initially responsive state (GLC14) to increased drug resistance for doxorubicin, etoposide, melphalan and vinblastine (GLC19), allowing for a gene expression analysis of the molecular changes acquired during the relapse and complete treatment failure. In the present work we describe a comparative analysis of the overexpressed gene transcripts and molecular pathways expected to be linked to tumor progression and chemoresistance in this case of SCLC.

\section{Materials and Methods}

\subsection{Cells and Reagents}

GLC14 and GLC19 cell lines were obtained from Dr. Nina Pedersen from the Department of Radiation Biology, The Finsen Centre, National University Hospital, Copenhagen, Denmark. Cells were grown in RPMI-1640 bicarbonate medium (Seromed, Berlin, Germany) supplemented with $10 \%$ fetal bovine serum (Seromed), $4 \mathrm{mM}$ glutamine and antibiotics $(10 \times$ stock formulated to contain $\sim 5000$ units penicillin, $5 \mathrm{mg}$ streptomycin and $10 \mathrm{mg}$ neomycin $/ \mathrm{ml})$ under tissue culture conditions $\left(37^{\circ} \mathrm{C}, 5 \%\right.$ $\mathrm{CO}_{2}, 95 \%$ humidity) and checked for mycoplasma contamination (Mycoplasma PCR ELISA, Roche Diagnostics, Vienna, Austria). Cells grow as spheroids in suspension and were subcultures two times a week. Except where otherwise stated all reagents were from Sigma-Aldrich, St. Louis, MO, USA.

\subsection{Chemosensitivity Assay}

$1 \times 10^{4}$ cells in $100 \mu \mathrm{l}$ medium per well were distributed in 96-well microtiter plates (Greiner, Kremsmuenster, Austria) and the test compound added in another $100 \mu 1$. Cisplatin (initial concentration $20 \mu \mathrm{g} / \mathrm{ml}$ ) and solute con- trols were serially diluted in twofold steps in triplicate. The microtiter plates were incubated under tissue culture conditions for four days and cell viability was measured using a modified MTT (3-(4,5-dimethylthiazol-2-yl)-2,5-diphenyl-tetrazolium bromide) assay (EZ4U, Biomedica, Vienna, Austria). Optical density was measured at $450 \mathrm{~nm}$ using a microplate reader with an empty well as reference. Values obtained from control wells containing cells and media alone were set to $100 \%$ proliferation. 


\subsection{Gene Expression and Bioinformatic Analysis}

Cells were cultivated in $150 \mathrm{~cm}^{2}$ flasks in triplicate under tissue culture conditions, harvested and pellets of approximately $35 \times 10^{6}$ cells stored frozen at $-80^{\circ} \mathrm{C}$. Briefly, lysis with extraction buffer (4 M guanidine isothiocyanate, $0.5 \%$ sodium $\mathrm{N}$-lauroylsarcosinate, $10 \mathrm{mM}$ EDTA, $5 \mathrm{mM}$ sodium citrate, $100 \mu \mathrm{M} \beta$-mercaptoethanol) was performed at $4{ }^{\circ} \mathrm{C}$, and DNA and RNA of the lysates were separated by cesium trifluoroacetate ultracentrifugation. RNA was washed with ice-cold 96\% ethanol and dissolved in water. Measurements of the optical density at 260/280 nm proved content and purity of the RNA. Gene expression analysis was performed in duplicate using the Applied Biosystems Human Genome Survey Microarray V2.0 (Applied Biosystems, Foster City, CA, USA). Therefore, 2 - $5 \mu \mathrm{g}$ mRNA (20 - $50 \mu \mathrm{g}$ total RNA) were reverse transcribed to first-strand cDNA and labeled with digoxigenin-UTP according to the Applied Biosystems Chemiluminescent Reverse Transcription protocol. Hybridization of cDNA and microarray analysis was performed pursuant to the Applied Biosystems Chemiluminescence Detection Kit protocol and by use of the Applied Biosystems 1700 Chemiluminescent Microarray Analyzer. Data were processed by filtering and quantile normalization. Microarray probe identities were allocated to the respective gene designations using the microarray data information provided by Applied Biosystems. Significance Analysis of Microarrays (SAM) was used to identify differentially expressed genes in GLC19 and GLC14 cells with a false discovery rate of $12 \%$ [10]. Pathway analysis was carried out by help of the Reactome database available at http://www.reactome.org [11]. Statistical analysis was performed using two-tailed Student's t-test for normally distributed samples $(* p<0.05$ was regarded as statistically significant).

\section{Results}

During the course of treatment the primary SCLC, represented by the chemosensitive GLC14 cell line, acquired a resistant phenotype that is retained in form of the GLC19 SCLC cell line. Since for this patient therapy had not included platinum-based drugs, we tested both cell lines in an MTT assay for their sensitivity to cisplatin in the present study. Dose-response curves revealed $\mathrm{IC}_{50}$ values of $1.3 \pm 0.2$ and $0.5 \pm 0.09 \mu \mathrm{g} / \mathrm{ml}$ for GLC14 and GLC19, respectively.

Gene transcripts overexpressed in GLC19 in comparison to GLC14 cells are listed in Table 1. 440 transcripts were found to be upregulated more than 2.5-fold in GLC19 vs. GLC14 SCLC cells. They include a number of proteins involved in regulation of cell growth, differentiation and cell death/apoptosis. The most prominent adaptor proteins affected were nerve growth factor re- ceptor-associated protein (NGFRAP1/BEX3) and CDK5 regulatory subunit associated protein 1 (CDK5RAP1). Increased expression was detected for the protooncogens myc and fos, as well as for the growth factors neurotensin (NTS), midkine (MDK; neurite growth-promoting factor 2), platelet-derived growth factor D (PDGFD), serine/threonine protein kinase MST4 and Mps one binder kinase activators (MOBs). Transcripts for vasoactive intestinal peptide receptor 1 (VIPR1), fibroblast growth factor receptor 1 (FGFR1) and ERBB4 exhibited relative overexpression in GLC19 cells.

A second group of upregulated messengers comprised proteins involved in cell adhesion/cell membrane signal transduction including cell surface associated mucin 15 (MUC15), epithelial membrane protein 2 (EMP2), Pleckstrin homology domain-containing family $\mathrm{C}$ member (PLEKHC1), receptor for hyaluronic acid CD44, calcium-dependent cell-adhesion protein protocadherin 18 (PCDH18), claudin 1 (CLDN1), cwcv and kazal-like domains proteoglycan sparc/osteonectin (SPOCK) and the neural cell adhesion molecule 2 (NCAM2). Increased transcription of glutathione S-transferases glutathione S-transferase theta 1 (GSTT1), glutathione Stransferase alpha 4 (GSTA4), microsomal glutathione S-transferase 2 (MGST2), and thioredoxin interacting protein (TXNIP) function in elevated conjugation of reduced glutathione to a wide number of exogenous and endogenous hydrophobic electrophiles for elimination and in balancing the redox state, respectively.

Several mediators upregulated in GLC19 cells belong to the wingless-type MMTV integration site family (Wnt) and to Notch signaling pathways. They comprise secreted frizzled-related protein 1 (SFRP1), WNT5B, coactivator mastermind-like 2 (MAML2), transducin-like enhancer of split 2 (E(sp1)) homolog (TLE2) and the Notch receptor ligands jagged 1/2 (JAG1 and JAG2). Putative markers of CSCs overexpressed in GLC19 cells moreover include CD44, aldehyde dehydrogenase 1 family, member A1 (ALDH1A1) and the aldo-keto reductase family 1 members C1/13 (AKR1C1 and AKR1C13). Furthermore, several members of the MAGE family that may play a role in embryonal development, tumor transformation or aspects of tumor progression were elevated in GLC19 cells. 60 gene transcripts were downregulated more than 3-fold in GLC19 vs. GLC14 cells and include several metallothioneins (MTs; 1A, 1F, 1X, 3 and 4), tumor protein 53 inducible protein 11 (TP53I11), as well as members of the tubulin family (TUBA3, TUBB4) and tubulin folding cofactor A (TBCA; data not shown).

The expression data was further analyzed for overrepresented pathways with help of the Reactome software (www.reactome.org) and the significant results are listed in Table 2. Increases in sphingolipid metabolism, expression of mediators of neuronal, Notch, nitric oxide and 
Table 1. Gene transcripts overexpressed in GLC19 vs GLC14.

\begin{tabular}{|c|c|c|c|}
\hline Identity (ID) & ID & Expression ratio & Gene symbol \\
\hline Applied Biosystems & Celera Genomics & GLC19/GLC14 & \\
\hline \multicolumn{4}{|c|}{ Growth regulators/factors/factor receptors } \\
\hline 210438 & hCG1992111 & 573.5 & NGFRAP1 \\
\hline 132666 & hCG15917.3 & 504.9 & MYC \\
\hline 160562 & hCG21128.4 & 150.2 & NTS \\
\hline 209085 & hCG18178.2 & 139.1 & POF1B \\
\hline 115497 & hCG2039648.2 & 90.9 & CDK5RAP1 \\
\hline 112198 & hCG1783468.2 & 67.8 & MST4 \\
\hline 128869 & hCG32359.4 & 24.3 & MOB \\
\hline 159434 & hCG28852.3 & 12.8 & VIPR1 \\
\hline 221237 & hCG23105.3 & 9.5 & FGFR1 \\
\hline 131372 & hCG28052.3 & 7.2 & GUCY1B3 \\
\hline 170165 & hCG28051.3 & 3.2 & GUCY1A3 \\
\hline 205128 & hCG22355.3 & 6.5 & FOS \\
\hline 111257 & hCG25765.4 & 4.4 & MDK \\
\hline 192974 & hCG2012284 & 3.5 & ERBB4 \\
\hline 108301 & hCG40536.2 & 3.9 & PDGFD \\
\hline 186955 & hCG2039636 & 2.7 & SDF2 \\
\hline \multicolumn{4}{|l|}{ Cell adhesion } \\
\hline 167910 & hCG27270.4 & 303.2 & MUC15 \\
\hline 173909 & hCG14644.2 & 41.8 & EMP2 \\
\hline 183236 & hCG20966.3 & 37.8 & PLEKHC1 \\
\hline 133604 & hCG1811182.2 & 13.6 & CD44 \\
\hline 199474 & hCG16335.3 & 10.8 & PCDH18 \\
\hline 152957 & hCG17574.3 & 6.4 & CLDN1 \\
\hline 125730 & hCG39479.3 & 4.4 & SPOCK \\
\hline 199050 & hCG2007901.1 & 3.4 & NCAM2 \\
\hline \multicolumn{4}{|l|}{ Glutathione metabolism } \\
\hline 118930 & hCG37372.3 & 6.9 & TXNIP \\
\hline 163516 & hCG39955.5 & 3.2 & GSTT1 \\
\hline 180333 & hCG37948.3 & 2.6 & MGST2 \\
\hline 173212 & hCG21940.3 & 2.5 & GSTA4 \\
\hline \multicolumn{4}{|l|}{ Wnt-Notch signaling pathways } \\
\hline 143998 & NM_003012.2 & 51.4 & SFRP1 \\
\hline 172164 & hCG24219.3 & 6.7 & WNT5B \\
\hline 167731 & hCG2043008 & 4.9 & MAML2 \\
\hline 141263 & hCG2039528 & 3.12 & TLE2 \\
\hline 123012 & hCG38131.3 & 3.1 & JAG1 \\
\hline 184984 & hCG1748359. & 2.8 & JAG2 \\
\hline \multicolumn{4}{|l|}{ MAGE } \\
\hline 159590 & hCG38709.2 & 32.6 & MAGEA4 \\
\hline 189042 & hCG1644199.2 & 15.8 & MAGEA9 \\
\hline 181315 & hCG38711.2 & 6.1 & MAGEA10 \\
\hline \multicolumn{4}{|l|}{ Putative stem cell markers } \\
\hline 162248 & hCG17306.2 & 8.6 & ALDH1A1 \\
\hline 122158 & hCG2017792 & 5.9 & AKR1C1 \\
\hline 129282 & hCG19343.4 & 4.9 & AKR1C13 \\
\hline
\end{tabular}

Table includes the Applied Biosystems identity number, human Celera Genomics clone designation, relative overexpression ratio and gene symbol. Transcripts are grouped according to their main cellular functions. 
Table 2. Cellular pathways overrepresented in GLC19 vs GLC14 SCLC cells.

\begin{tabular}{ccl}
\hline p-value & Identifier & Name of event \\
\hline 0.001 & REACT_19323 & Sphingolipid metabolism \\
0.004 & REACT_13685 & Neuronal system \\
0.005 & REACT_118859 & Signaling by Notch \\
0.008 & REACT_23862 & Nitric oxide stimulates guanylate cyclase \\
0.018 & REACT_24989 & ATPase type IV transporters - phospholipid transfer \\
0.027 & REACT_75770 & Voltage gated potassium channels \\
0.03 & REACT_15518 & Transmembrane transport of small molecules \\
0.048 & REACT_21310 & Phospholipase C-mediated cascade \\
\hline
\end{tabular}

The list of overexpressed gene transcripts in GLC19 vs GLC14 was used to identify overrepresented cellular pathways.

phospolipase $\mathrm{C}$ signaling, as well as of ion/small molecules/phospolipid membrane transporters were observed.

\section{Discussion}

The GLC14 cell line was established using a biopsy from a right supraclavicular node of a 55-year-old woman with extended SCLC. Treatment was started with cyclophosphamide, doxorubicin and etoposide (CDE), which resulted in a complete response [9]. After seven months tumor recurrence was detected in chest X-ray and reinduction chemotherapy with CDE led to a partial response. Radiotherapy was given to the left hilar region and mediastinum and led to a normal chest X-ray, before SCLC reappeared in the left upper lobe of the lung. At this stage another cell line (GLC19) was established from a biopsy. The tumor progressed despite two additional cycles of $\mathrm{CDE}$ and the patient died after discontinuation of therapy, 17 months after first presentation. The electron microscopic appearance of all cell lines was compatible with SCLC of the "classic type". Additionally, dense core vesicles were present in moderate amounts in all cell lines, whereas desmosomes with associated tonofilaments were identified in GLC19 cells only. The $\mathrm{IC}_{50}$ for doxorubicin and etoposide increased 1.7-fold from 0.44 for GLC14 to $0.75 \mathrm{mM}$ for GLC19 and 5.5-fold from 4.5 for GLC14 to $24.8 \mathrm{ng} / \mathrm{ml}$ for GLC19, respectively. Similarly, $\mathrm{IC}_{50}$ values for GLC14 and GLC19 for vinblastine were 445 and $1936 \mathrm{ng} / \mathrm{ml}$, for vincristine, 145.7 and $179.9 \mathrm{ng} / \mathrm{ml}$ and for actinomycin, 0.084 and $0.337 \mu \mathrm{g} / \mathrm{ml}$, revealing an increased drug resistance for GLC19 cells for doxorubicin, etoposide, Vinca alkaloids and actinomycin. Whereas in RPMI-1640 with 10\% heat-inactivated fetal calf serum GLC14 cells grew almost completely attached to the culture flask, GLC19 cells formed floating aggregates and exhibited a prolonged doubling time of $44 \mathrm{hrs}$ compared to $26 \mathrm{hrs}$ for GLC14 cells [12]. Cisplatin was not used for treatment of this patient and we found a slight decrease of the chemosensitivity for this drug in GLC19 vs GLC14 cells in good agreement with a previous report [12].
In order to detect global changes in gene expression linked to SCLC tumor progression and chemoresistance, we compared gene transcripts of GLC14 and GLC19 cells using analysis by the Applied Biosystems Human Genome Survey Microarrays V2.0. Approximately 440 genes were more than 2.5-fold overexpressed in GLC19 cells and 100 genes were more than 3-fold downregulated in this cell line compared to GLC14 cells. Of the 440 upregulated transcripts, approximately 300 could be assigned to annotated genes. 40 of these with strongest elevations and highest relevance for the SCLC phenotype under investigation are listed in Table 1. 37/60 downregulated transcripts could be traced to annotated genes.

A panel of genes upregulated in GLC19 vs. GLC14 cells are involved in regulation of cell growth, differentiation and cell death/apoptosis. Overexpression of NGFRAP1/BEX3, a p75NTR (low-affinity neurotrophin receptor p75)-associated cell death executor initially cloned from a human ovarian granulosa cell cDNA library as an unknown protein termed pHGR74, was likewise reported by Lawson et al. and constituted the most upregulated gene in our data set $[13,14]$. BEX3 belongs to a family of genes, including BEX1, NGFRAP1 (alias BEX3), BEXL1 (alias BEX4), and NGFRAP1L1 (alias BEX5) [15]. Both BEX1 and NGFRAP1 interact with p75NTR and modulate NGF signaling through NF- $\mathrm{kB}$ to regulate cell cycle, apoptosis and differentiation in neural tissues [16]. Enforced expression of NGFRAP1 in Chinese hamster ovary cells and MDA-MB-231 human breast cancer cells had little effect on the growth of the cells in vitro, while cellular growth was dramatically suppressed in vivo [17]. Furthermore, the cells stably transfected with NGFRAP1 did not respond neither to NGF nor TNF. These findings may be linked to the prolonged doubling time of GLC19 vs. GLC14 cells. In breast cancer cells, NGF inhibits ceramide-homolog-induced apoptosis through binding of p75NTR and NF- $\mathrm{KB}$ activation via BEX2. Additionally, overexpression of NGFRAP1 enhances the antiproliferative effect of tamoxifen at pharmacological dose. Furthermore, NGFR-AP1 was reported to consti- 
tute a marker of androgen-dependent primary prostate cancer [18]. A second cell cycle regulator, namely CDK5RAP1, specifically inhibits CDK5 activation by CDK5R1, which is the catalytic subunit of neuronal Cdc2-like kinase (Nclk) involved inneuronal cell differentiation, and apoptosis [19].

Increased expression was detected for the protooncogens myc and fos, as well for the growth factors neurotensin (NTS), MDK, PDGFD, serine/threonine protein kinase MST4 and Mps one binder kinase activators (MOBs). Upregulation of the expression of myc in GLC19 was previously described by Kok et al. [20]. Amplification and overexpression of the MYC family of oncogenes, MYC (c-Myc), MYCN (N-Myc) or MYCL1 (L-Myc), occurs in $18 \%-31 \%$ of SCLCs, and is more common in chemorefractory disease [21]. Transcripts for VIPR1, FGFR1 and ERBB4 exhibited relative overexpression in GLC19 cells, which indicates cellular responses to their cognate growth factors VIP, FGF and EGF, respectively. VIP in SCLC is involved in upregulation of VEGF via fos, and FGFs as well as EGF are support the proliferation of CSCs under serum-depleted conditions $[22,23]$. In general, neuropeptides can function as autocrine growth factors in cancer cells or modulate the microenvironment [24,25].

GLC19 cells overexpress WNT5B, a ligand for members of the frizzled family of seven transmembrane receptors, SFRP1 which functions as modulator of Wnt signaling through direct interaction with Wnts, and TLE2, which inhibits the transcriptional activation mediated by $\beta$-catenin (CTNNB1) and TCF/LEF family members in Wnt signaling [26]. The Notch pathway powerfully influences stem cell maintenance, development and cell fate and is increasingly recognized for the key roles it plays in cancer [27]. Notch promotes cell survival, angiogenesis and resistance in numerous cancers, making it a promising target for cancer therapy. In respect to the Notch signaling pathway, MAML2, which acts as a transcriptional coactivator for Notch proteins as well as increased expression of JAG1 and JAG2, that are ligands for multiple Notch receptors are distinctive characteristics of GLC14 and GLC19 cells. Notch activation induces the transcription of both subunits of the soluble guanylyl cyclase (sGC) heterodimer, namely GUCY1A3 and GUCY1B3, which form the nitric oxide receptor [28]. Additionally, the core stem cell signaling networks, such as the Wnt, Notch and Hedgehog pathways, also critically regulate the self-renewal and survival of CSCs [29].

A number of upregulated transcripts in GLC19 cells comprise genes involved in cell adhesion/cell membrane signaling in accordance with the exclusive finding of desmosomes in GLC19 exclusively in this series of SCLC lines [9]. CD44 cell-surface glycoprotein, which represents the receptor for hyaluronic acid (HA), is in- volved in cell-cell interactions, cell adhesion and migration [30]. It mediates cell-cell and cell-matrix interacttions through its affinity for HA and possibly also for other ligands such as osteopontin, collagens, and matrix metalloproteinases (MMPs). Furthermore, CD44 has been defined as a CIC marker in many tumor entities, promoting epithelial-to-mesenchymal transition (EMT), motility, endothelial cell and niche adhesion, thereby contributing to niche generation and modulation [31]. MUC15 may play a role in cell adhesion to the extracellular matrix (ECM) and promotes the oncogenic potential of human colon cancer cells [32]. The upregulated transcripts PLEKHC1, SPOCK, CLDN1 and EMP2 connect ECM adhesion sites to the actin cytoskeleton, mediate calcium-independent cell-adhesion and regulate the surface display and signaling from selected integrin pairs, respectively [33-35]. NCAM2 is a close homolog of neural cell adhesion molecule NCAM1 (CD56), which is expressed in several types of tumors, constitutes a diagnostic marker of SCLCs, and is associated with tumorigenesis in salivary gland tumors [36]. Compared with NCAM1, the function of NCAM2 in tumors, such as prostate and breast cancer, is unknown, but may represent a therapeutic target [37].

Many principal antitumor agents, such as platinum complexes and doxorubicin are electrophiles or redox-active agents and may preferentially react with sulfhydryl groups [38]. The elevated amount of glutathione (GSH) and the increased activity of GSTs can explain the resistance to alkylating agents and other drugs that act by releasing free radicals. Our finding of increased transcription of the GSTs GSTT1, GSTA4 and MGST2 in GLC19 cells is in good agreement with data published in a previous analysis of its mechanisms of resistance [10]. The GSH level of GLC19 and global GST activity were significantly increased compared to GLC14, whereas the amount of total sulfhydryl groups remained the same and, furthermore, L-buthionine-sulfoximine (BSO), which depletes the cellular GSH pool, increased both doxorubicin- and cisplatin-induced cytotoxicity. This seems to constitute an important mechanism of chemoresistance for GLC19 cells, since other effectors like P-gp are absent in GLC19 [10].

ALDH1A1 was expressed at low level in GLC14 cells with the amount rising in the GLC19 cells. This gene is used as a CSC marker and is also associated with cyclophosphamide resistance [39-41]. The AKR1C1/13 genes encode members of the aldo/keto reductase super-family, that consists of more than 40 known enzymes, which catalyze the conversion of aldehyde and ketone moieties of metabolites and xenobiotics to corresponding alcohols by utilizing NADH and/or NADPH as cofactors. $\mathrm{AKR} 1 \mathrm{C} 1$ and $\mathrm{C} 2$ were found as genes linked to poor clinical prognosis in non-small cell lung cancer (NSCLC) 
following characterization of elevated transcripts of the A549 CSCs Hoechst 33342-positive side population [42]. AKR1C13 transcription was increased in leukemia cells following transcription factor Meis1-triggered induction of a CSC phenotype [43]. In our data set MEIS1 (hCG1818196.1) was upregulated 3.5-fold in GLC19 compared to GLC14 cells. A family of cancer-related molecules are the MAGEs that are in normal tissues exclusively found in testis and often highly expressed in cancer cell lines and tumors. They are targets for many studies on immunotherapy for melanoma; however, since tumor xenografts of SCLC cell lines had a markedly lower amount of MAGEs, expression of these genes seems to be activated in tissue culture only and therefore not to represent a promising target for this tumor entity [44]. Lawson et al. analyzed the GLC14/16/19 series of SCLC cell lines using the Illumina Human WGv3.0 Expression BeadChip microarray and identified polymerase B (POLB) and NKX2.2 as transcripts linked to resistance to etoposide [14]. Of the selected unique gene transcripts that were upregulated in GLC19 compared to GLC14 as published by Lawson et al. 18/23 probes were as well found. Additionally, we found overexpression of growth factors and their cognate receptors that was not reported before.

Downregulationed gene expression in GLC19 in comparison to GLC14 cells was detected for a comparatively low number of approximately 60 transcripts. Surprisingly, GLC14 cells expressed significant amounts of MTs (1A, 1F, 1X, 3 and 4), which showed lower levels of transcripts after several cycles of therapy in GLC19 cells. Presence of these MTs in the chemonaive GLC14 cells is could be explained by a smoking history of the affected patient, which is known to be associated with increased expression of these heavy metal-binding proteins [45]. The tumor suppressor p53 regulates the expression of various genes that promote apoptosis. TP53I11 is a direct target gene of p53 and was markedly upregulated in arsenic trioxide-induced apoptosis and, thus, downregulation of this protein may impede druginduced apoptosis [46]. Downregulation of the transcription of TUBA3, TUBB4 and tubulin folding cofactor A TBCA seems to be a consequence of the crossresistance of GLC19 cells to Vinca alkaloids [47].

Sphingolipid metabolism constituted the most overrepresented pathway in GLC19 vs. GLC14 cells. Sphingolipids are known to play important roles in the regulation of cell proliferation, response to chemo-therapeutic agents, and/or prevention of cancer. [48,49]. Bioactive sphingolipids like ceramide, sphingosine 1-phosphate and globotriaosylceramide initiate and process cellular signaling to alter cell behaviour to respond immediately to oncogenic stress or treatment challenges. More than ten sphingolipids and glycosphingolipids selectively me- diate the expression of approximately 50 genes encoding c-myc, p21, c-fos, telomerase reverse transcriptase, caspase-9, Bcl-x, cyclooxygenase-2, MMPs, integrins, Oct-4, glucosylceramide synthase and P-gp [50]. Through diverse functions of these genes, sphingolipids enduringly affect cellular processes of mitosis, apoptosis, migration, stemness of CSCs and cellular resistance to therapies. Mechanistic studies indicate that sphingolipids regulate gene expression by modulation of phosphorylation and acetylation of proteins that serve as transcription factors ( $\beta$-catenin, $\mathrm{Sp} 1$ ), repressor of transcription (histone $\mathrm{H} 3$ ) and regulator (SRp30a) of RNA splicing.

In conclusion, GLC19 cells are distinguished from GLC14 by a reduced growth rate, formation of multicellular spheroids, expression of CD44, increased expression of components of the Wnt and Notch signaling pathways, as well as receptors of the growth factors FGFs and EGF and the elevated transcription of the CSC markers ALDH1A1 and AKR1C1/13. Although the microarray results need to be confirmed by PCR, our findings are compatible with the typical signature established for CICs or CSCs and different tumor entities [51,52]. Characterization of CICs in SCLC is being ongoing and increased expression of ABCG2, FGF1, IGF1, MYC, SOX1/2, WNT1, as well as genes involved in angiogenesis, Notch and Hedgehog pathways was reported for this type of tumor cells that comprise a fraction of less than one percent in SCLC cell lines [53]. The gene expression profile of GLC19 cells indicates a therapy-induced increase of typical stem cell markers in vivo and enhanced cell adhesion and formation of spheroids may constitute an important part in the acquisition of chemoresistance through a reduced access of chemotherapeutic drugs [54].

\section{Acknowledgements}

This work was funded by the "Medical Scientific Fund of the Mayor of the City of Vienna", project number 11016. We wish to thank the team of the "Oncology Lab" of the University Clinic of Gynecology, Medical University of Vienna, Austria, for help with the microarry experiments.

\section{REFERENCES}

[1] D. R. Youlden, S. M. Cramb and P. D. Baade, "The International Epidemiology of Lung Cancer: Geographical Distribution and Secular Trends," Journal of Thoracic Oncology, Vol. 3, No. 8, 2008, pp. 819-831. doi:10.1097/JTO.0b013e31818020eb

[2] S. G. Spiro and G. A. Silvestri, "One Hundred Years of Lung Cancer," American Journal of Respiratory Critical Care Medicine, Vol. 172, No. 5, 2005, pp. 523-529. doi:10.1164/rccm.200504-531OE

[3] R. Califano, A. Z. Abidin, R. Peck, C. Faivre-Finn and P. 
Lorigan, "Management of Small Cell Lung Cancer: Recent Developments for Optimal Care," Drugs, Vol. 72, No. 4, 2012, pp. 471-490. doi:10.2165/11597640-000000000-00000

[4] R. Govindan, N. Page, D. Morgensztern, et al., "Changing Epidemiology of Small-Cell Lung Cancer in the United States over the Last 30 Years: Analysis of the Surveillance, Epidemiologic, and End Results Database," Journal of Clinical Oncology, Vol. 24, 2006, pp. 45394544. doi:10.1200/JCO.2005.04.4859

[5] W. N. William and B. S. Glisson, "Novel Strategies for the Treatment of Small-Cell Lung Carcinoma, "Nature Reviews Clinical Oncology, Vol. 8, No. 10, 2011, pp. 611-619. doi:10.1038/nrclinonc.2011.90

[6] Y. Agra, M. Pelayo, M. Sacristan, et al., "Chemotherapy versus Best Supportive Care for Extensive Small Cell Lung Cancer," Cochrane Database Systematic Review, Vol. 4, 2003, Article ID: CD001990.

[7] M. Takada, M. Fukuoka, M. Kawahara, et al., "Phase III Study of Concurrent versus Sequential Thoracic Radiotherapy in Combination with Cisplatin and Etoposide for Limited-Stage Small-Cell Lung Cancer," Journal of Clinical Oncology, Vol. 20, 2002, pp. 3054-3060. doi:10.1200/JCO.2002.12.071

[8] B. Fischer and A. Arcaro, "Current Status of Clinical Trials for Small Cell Lung Cancer," Review of Recent Clinical Trials, Vol. 3, 2008, pp. 40-61. doi: $10.2174 / 157488708783330503$

[9] H. H. Berendsen, L. de Leij, E. G. de Vries, G. Mesander, N. H. Mulder, B. de Jong, C. H. Buys, P. E. Postmus, S. Poppema, H. J. Sluiter, et al., "Characterization of Three Small Cell Lung Cancer Cell Lines Established from One Patient during Longitudinal Follow-Up," Cancer Research, Vol. 48, No. 23, 1988, pp. 6891-6899.

[10] V. G. Tusher, R. Tibshirani and G. Chu, "Significance Analysis of Microarrays Applied to the Ionizing Radiation Response," Proceedings of the National Academy of Science of the USA, Vol. 98, No. 9, 2001, pp. 5116-5121. doi:10.1073/pnas.091062498

[11] D. Croft, G. O'Kelly, G. Wu, R. Haw, M. Gillespie, L. Matthews, M. Caudy, P. Garapati, G. Gopinath, B. Jassal, S. Jupe, I. Kalatskaya, S. Mahajan, B. May, N. Ndegwa, E. Schmidt, V. Shamovsky, C. Yung, E. Birney, H. Hermjakob, P. D'Eustachio and L. Stein, "Reactome: A Database of Reactions, Pathways and Biological Processes," Nucleic Acids Research, Vol. 39, 2011, pp. D691D697. doi:10.1093/nar/gkq1018

[12] E. G. de Vries, C. Meijer, H. Timmer-Bosscha, H. H. Berendsen, L. de Leij, R. J. Scheper and N. H. Mulder, "Resistance Mechanisms in Three Human Small Cell Lung Cancer Cell Lines Established from One Patient during Clinical Follow-Up," Cancer Research, Vol. 49, No. 15, 1989, pp. 4175-4178.

[13] G. Rapp, J. Freudenstein, J. Klaudiny, J. Mucha, F. Wempe, M. Zimmer and K. H. Scheit, "Characterization of Three Abundant mRNAs from Human Ovarian Granulosa Cells," DNA Cell Biology, Vol. 9, No. 7, 1990, pp. 479-485.doi:10.1089/dna.1990.9.479

[14] M. H. Lawson, N. M. Cummings, D. M. Rassl, R. Russell,
J. D. Brenton, R. C. Rintoul and G. Murphy, "Two Novel Determinants of Etoposide Resistance in Small Cell Lung Cancer," Cancer Research, Vol. 71, No. 14, 2011, pp. 4877-4887. doi:10.1158/0008-5472.CAN-11-0080

[15] A. Naderi, A. E. Teschendorff, J. Beigel, M. Cariati, I. O. Ellis, J. D. Brenton and C. Caldas, "BEX2 Is Overexpressed in a Subset of Primary Breast Cancers and Mediates Nerve Growth Factor/Nuclear Factor-KappaB Inhibition of Apoptosis in Breast Cancer Cell Lines," Cancer Research, Vol. 67, No. 14, 2007, pp. 6725-6736. doi:10.1158/0008-5472.CAN-06-4394

[16] N. H. Molloy, D. E. Read and A. M. Gorman, "Nerve Growth Factor in Cancer Cell Death and Survival," Cancers, Vol. 3, 2011, pp. 510-530. doi:10.3390/cancers3010510

[17] X. Tong, D. Xie, W. Roth, J. Reed, H. P. Koeffler, "NADE (p75NTR-Associated Cell Death Executor) Suppress Cellular Growth in Vivo," International Journal of Oncology, Vol. 22, 2003, pp. 1357-1362.

[18] T. L. Romanuik, T. Ueda, N. Le, S. Haile, T. M. Yong, T. Thomson, R. L. Vessella and M. D. Sadar, "NGFRAP1 Novel Biomarkers for Prostate Cancer Including Noncoding Transcripts," American Journal of Pathology, Vol. 175, No. 6, 2006, pp. 2264-2276. doi:10.2353/ajpath.2009.080868

[19] Y. P. Ching, A. S. Pang, W. H. Lam, R. Z. Qi and J. H. Wang, "Identification of a Neuronal Cdk5 ActivatorBinding Protein as Cdk5 Inhibitor," Journal of Biological Chemistry, Vol. 277, No. 18, 2002, pp. 15237-15240. doi:10.1074/jbc.C200032200

[20] K. Kok, J. Osinga, D. C. Schotanus, H. H. Berendsen, L. F. de Leij and C. H. Buys, "Amplification and Expression of Different Myc-Family Genes in a Tumor Specimen and 3 Cell Lines Derived from One Small-Cell Lung Cancer Patient during Longitudinal Follow-Up," International Journal of Cancer, Vol. 44, No. 1, 1989, pp. 75-78. doi:10.1002/ijc.2910440114

[21] I. I. Wistuba, A. F. Gazdar and J. D. Minna, "Molecular Genetics of Small Cell Lung Carcinoma," Seminars in Oncology, Vol. 28, No. 2-4, 2001, pp. 3-13.

[22] Z. Zhao, Q. Cheng, X. Li, X.Wang and K. Liu, “c-fos Antisense Oligodeoxynucleotide Reduces VIP-Induced Upregulation of VEGF Expression in Small Cell Lung Cancer Cells," Chinese Journal of Lung Cancer, Vol. 9, No. 4, 2006, pp. 312-315.

[23] C. A. Gilbert and A. H. Ross, "Cancer Stem Cells: Cell Culture, Markers and Targets for New Therapies," Journal of Cellular Biochemistry, Vol. 108, No. 5, 2009, pp. 1031-1038. doi:10.1002/jcb.22350

[24] T. W. Moody, D. Chan, J. Fahrenkrug and R. T. Jensen, "Neuropeptides as Autocrine Growth Factors in Cancer Cells," Current Pharmaceutical Design, Vol. 9, No. 6, 2003, pp. 495-509. doi:10.2174/1381612033391621

[25] U. Olszewski and G. Hamilton, "Neurotensin Signaling Induces Intracellular Alkalinization and Interleukin-8 Expression in Human Pancreatic Cancer Cells," Molecular Oncology, Vol. 3, 2009, pp. 204-213. doi:10.1016/j.molonc.2009.01.006

[26] H. Yao, E. Ashihara and T. Maekawa, "Targeting the 
Wnt $/ \beta$-Catenin Signaling Pathway in Human Cancers," Expert Opinion on Therapeutic Targets, Vol. 15, No. 7, 2011, pp. 873-887. doi: $10.1517 / 14728222.2011 .577418$

[27] B. Purow, "Notch Inhibition as a Promising New Approach to Cancer Therapy," Advances in Experimental Medicine and Biology, Vol. 727, 2012, pp. 305-319. doi:10.1007/978-1-4614-0899-4 23

[28] A. C. Chang, Y. Fu, V. C. Garside, K. Niessen, L. Chang, M. Fuller, A. Setiadi, J. Smrz, A. Kyle, A. Minchinton, M. Marra, P. A. Hoodless and A. Karsan, "Notch Initiates the Endothelial-to-Mesenchymal Transition in the Atrioventricular Canal through Autocrine Activation of Soluble Guanylyl Cyclase," Developmental Cell, Vol. 21, No. 2, 2011, pp. 288-300. doi:10.1016/j.devcel.2011.06.022

[29] J. Wang, B. A. Sullenger and J. N. Rich, "Notch Signaling in Cancer Stem Cells," Advances in Experimental Medicine and Biology, Vol. 727, 2012, pp. 174-185. doi:10.1007/978-1-4614-0899-4 13

[30] J. Lesley, R. Hyman and P. W. Kincade, "CD44 and Its Interaction with Extracellular Matrix," Advances in Immunology, Vol. 54, 1993, pp. 271-335. doi:10.1016/S0065-2776(08)60537-4

[31] M. Zöller, "CD44: Can a Cancer-Initiating Cell Profit from an Abundantly Expressed Molecule?" Nature Reviews Cancer, Vol. 11, No. 4, 2011, pp. 254-267. doi:10.1038/nrc3023

[32] J. Huang, M. I. Che, Y. T. Huang, M. K. Shyu, Y. M. Huang, Y. M. Wu, W. C. Lin, P. H. Huang, J. T. Liang, P. H. Lee and M. C. Huang, "Overexpression of MUC15 Activates Extracellular Signal-Regulated Kinase 1/2 and Promotes the Oncogenic Potential of Human Colon Cancer Cells," Carcinogenesis, Vol. 30, No. 8, 2009, pp. 1452-1458. doi:10.1093/carcin/bgp137

[33] F. Siddiq, F. H. Sarkar, A. Wali, H. I. Pass and F. Lonardo, "Increased Osteonectin Expression Is Associated with Malignant Transformation and Tumor Associated Fibrosis in the Lung," Lung Cancer, Vol. 45, No. 2, 2004, pp. 197-205.doi:10.1016/j.lungcan.2004.01.020

[34] M. Fu, E. L. Maresh, R. A. Soslow, M. Alavi, V. Mah, Q. Zhou, A. Iasonos, L. Goodglick, L. K. Gordon, J. Braun and M. Wadehra, "Epithelial Membrane Protein-2 Is a Novel Therapeutic Target in Ovarian Cancer," Clinical Cancer Research, Vol. 16, No. 15, 2010, pp. 3954-3963. doi:10.1158/1078-0432.CCR-10-0368

[35] R. O. Hynes, "The Extracellular Matrix: Not Just Pretty Fibrils," Science, Vol. 326, No. 5957, 2009, pp. 12161219. doi:10.1126/science. 1176009

[36] M. Jensen and F. Berthold, "Targeting the Neural Cell Adhesion Molecule in Cancer," Cancer Letters, Vol. 258, 2007, pp. 9-21. doi:10.1016/j.canlet.2007.09.004

[37] S. Takahashi, K. Kato, K. Nakamura, R. Nakano, K. Kubota and H. Hamada, "Neural Cell Adhesion Molecule 2 as a Target Molecule for Prostate and Breast Cancer Gene Therapy," Cancer Science, Vol. 102, No. 4, 2011, pp. 808-814. doi:10.1111/j.1349-7006.2011.01855.x

[38] M. Namdarghanbari, W. Wobig, S. Krezoski, N. M. Tabatabai and D. H. Petering, "Mammalian Metallothionein in Toxicology, Cancer, and Cancer Chemotherapy,"
Journal of Biologic Inorganic Chemistry, Vol. 16, No. 7, 2011, pp. 1087-1101.

[39] J. S. Moreb, "Aldehyde Dehydrogenase as a Marker for Stem Cells," Current Stem Cell Research and Therapy, Vol. 3, No. 4, 2008, pp. 237-246. doi: $10.2174 / 157488808786734006$

[40] J. Hilton, "Role of Aldehyde Dehydrogenase in Cyclophosphamide-Resistant L1210 Leukemia," Cancer Research, Vol. 44, No. 11, 1984, pp. 5156-5160.

[41] X. Li, L. Wan, J. Geng, C. L. W and X. Bai, "Aldehyde Dehydrogenase 1A1 Possesses Stem-Like Properties and Predicts Lung Cancer Patient Outcome," Journal of Thoracic Oncology, 2012, in Press. doi:10.1097/JTO.0b013e318257cc6d

[42] D. C. Seo, J. M. Sung, H. J. Cho, H. Yi, K. H. Seo, I. S. Choi, D. K. Kim, J. S. Kim, A. El-Aty and H. C. Shin, "Gene Expression Profiling of Cancer Stem Cell in $\mathrm{Hu}-$ man Lung Adenocarcinoma A549 Cells," Molecular Cancer, Vol. 6, No. 75, 2007. doi:10.1186/1476-4598-6-75

[43] G. G. Wang, M. P. Pasillas and M. P. Kamps, "Meis1 Programs Transcription of FLT3 and Cancer Stem Cell Character, Using a Mechanism That Requires Interaction with $\mathrm{Pbx}$ and a Novel Function of the Meis1 C-Terminus," Blood, Vol. 106, No. 1, 2005, pp. 254-264. doi:10.1182/blood-2004-12-4664

[44] N. Pedersen, S. Mortensen, S. B. Sørensen, M. W. Pedersen, K. Rieneck, L. F. Bovin and H. S. Poulsen, "Transcriptional Gene Expression Profiling of Small Cell Lung Cancer Cells," Cancer Research, Vol. 63, No. 8, 2003, pp. 1943-1953.

[45] Koomägi, G. Stammler, C. Manegold, J. Mattern and M. Volm, "Expression of Resistance-Related Proteins in Tumoral and Peritumoral Tissues of Patients with Lung Cancer," Cancer Letters, Vol. 110, No. 1-2, 1996, pp. 129-136. doi:10.1016/S0304-3835(96)04471-0

[46] X. Q. Liang, E. H. Cao, Y. Zhang and J. F. Qin, “A P53 Target Gene, PIG11, Contributes to Chemosensitivity of Cells to Arsenic Trioxide," FEBS Letters, Vol. 569, No. 1-3, 2004, pp. 94-98. doi:10.1016/j.febslet.2004.05.057

[47] A. Cormier, M. Knossow, C. Wang and B. Gigant, "The Binding of Vinca Domain Agents to Tubulin: Structural and Biochemical Studies," Methods in Cell Biology, Vol. 95, 2010, pp. 373-390. doi:10.1016/S0091-679X(10)95020-6

[48] B. Ogretmen, "Sphingolipids in Cancer: Regulation of Pathogenesis and Therapy," FEBS Letters, Vol. 580, No. 23, 2006, pp. 5467-5476. doi:10.1016/j.febslet.2006.08.052

[49] G. A. Patwardhan and Y. Y. Liu, "Sphingolipids and Expression Regulation of Genes in Cancer," Progess in Lipid Research, Vol. 50, No. 1, 2011, pp. 104-114. doi:10.1016/j.plipres.2010.10.003

[50] M. Kohno, M. L. Momoi, J. H. Oo, Y. M. Paik, K. Lee, Venkataraman, et al., "Intracellular Role for Sphingosine Kinase 1 in Intestinal Adenoma Cell Proliferation," Molecular Cell Biology, Vol. 26, 2006, pp. 7211-7223. doi:10.1128/MCB.02341-05 
[51] B. Malik and D. Nie, "Cancer Stem Cells and Resistance to Chemo and Radio Therapy," Frontiers of Bioscience, Vol. 4, 2012, pp. 2142-2149. doi:10.2741/E531

[52] M. R. García Campelo, G. A. Curbera, G. Aparicio Gallego, E. G. Pulido and L. M. A. Aparicio, "Stem Cell and Lung Cancer Development: Blaming the Wnt, Hh and Notch Signalling Pathway," Clinical and Translational Oncology, Vol. 13, No. 2, 2011, pp. 77-83. doi:10.1007/s12094-011-0622-0

[53] C. D. Salcido, A. Larochelle, B. J. Taylor, C. E. Dunbar and L. Varticovski, "Molecular Characterisation of Side Population Cells with Cancer Stem Cell-Like Characteristics in Small-Cell Lung Cancer," British Journal of Cancer, Vol. 102, No. 11, 2010, pp. 1636-1644. doi:10.1038/sj.bjc. 6605668

[54] R. E. Durand and P. L. Olive, "Resistance of Tumor Cells to Chemo- and Radiotherapy Modulated by the ThreeDimensional Architecture of Solid Tumors and Spheroids," Methods in Cell Biology, Vol. 64, 2001, pp. 211233. doi:10.1016/S0091-679X(01)64015-9 